\title{
COVID-19-Induced Hyperleucocytosis in Chronic Lymphocytic Leukaemia
}

\author{
Balraj Singh, Sarah Ayad, Parminder Kaur, Ro-Jay Reid, Sachin Gupta, Michael Maroules \\ Saint Joseph's University Medical Center, Peterson, NJ, USA
}

Doi: 10.12890/2021_002348- European Journal of Case Reports in Internal Medicine - @ EFIM 2021

Received: 02/01/2021

Accepted: 08/02/2021

Published: 15/03/2021

How to cite this article: Singh B, Ayad S, Kaur P, Reid RJ, Gupta S, Maroules M. COVID-10-induced in chronic lymphocytic leukaemia. EJCRIM 2021;8: doi:10.12890/2021_002348

Conflicts of Interests: The Authors declare that there are no competing interests.

This article is licensed under a Commons Attribution Non-Commercial 4.0 License

\section{ABSTRACT}

Severe acute respiratory syndrome coronavirus 2 (SARS-CoV-2) is a novel coronavirus responsible for the current global pandemic, coronavirus disease 2019 (COVID-19). COVID-19 usually presents with respiratory symptoms but can affect multiple organ systems. A wide spectrum of complications can occur depending upon the comorbidities of patients. There is limited literature available regarding the presentation and outcome of COVID-19 in chronic lymphocytic leukaemia (CLL) patients. We report 2 cases of COVID-19-induced hyperleucocytosis (WBC count $>100,000 / \mu \mathrm{l}$ ) in CLL patients.

\section{LEARNING POINTS}

- Lymphopenia has been associated with severe disease and is a poor prognostic factor in COVID-19 infected patients; however, our cases

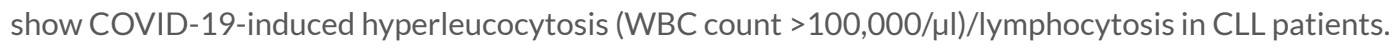

- Prior reports suggest that ibrutinib may have a protective effect against COVID-19 by decreasing inflammation and preventing progression to ARDS.

\section{KEYWORDS}

COVID-19, SARS-CoV-2, CLL, chronic lymphocytic leukaemia, lymphocytosis, leucocytosis, lymphopenia

\section{INTRODUCTION}

The pandemic caused by the novel severe acute respiratory syndrome coronavirus 2 (SARS-CoV-2), known as coronavirus disease 2019 (COVID-19), has contributed to a global health crisis and a magnitude of consequences. The virus has affected more than 93 million people and claimed the lives of more than 2 million individuals globally, according to the World Health Organization ${ }^{[1]}$. Risk factors associated with a poor outcome include advanced age, hypertension, diabetes, coronary artery disease and chronic kidney disease ${ }^{[2]}$. The clinical course of COVID-19 can range from mild respiratory symptoms to severe extensive lung damage and the development of acute respiratory distress syndrome (ARDS) along with multi-organ failure ${ }^{[3]}$.

A nationwide analysis by Liang et al. proposed that cancer patients may be at increased risk of developing COVID-19 and those with COVID-19 had a poor outcome compared to individuals without cancer [4]. We report a case series of 2 patients with chronic lymphocytic leukaemia (CLL) who were diagnosed with COVID-19 and developed hyperleucocytosis, as well as a review of the literature on the outcome and clinical course of COVID-19 amongst patients with CLL.

\section{CASE PRESENTATION 1}

The patient was an 81-year-old female with a past medical history of CLL (diagnosed in 2010), dyslipidaemia, hypothyroidism and hypertension who presented to the emergency room from a nursing home facility with a 1-week history of increasing fatigue, weakness, loss of appetite, dyspnoea on exertion and inability to perform daily activities. She denied any fever, nausea, vomiting or changes in urinary 
or bowel habits. The patient was diagnosed with Rai stage O CLL in 2010 with a baseline white blood cell (WBC) count of 60-70 K and was under observation. A nasopharyngeal swab tested positive for SARS-CoV-2 using the real-time reverse transcription-polymerase chain reaction (rRT-PCR) assay.

In the emergency department (ED), initial vital signs were significant for temperature $36.7^{\circ} \mathrm{C}$, heart rate 106 beats/minute, respiratory rate 20 breaths/minute, blood pressure 145/62 mmHg, oxygen saturation of $96 \%$ on room air. The physical examination was unremarkable. Initial laboratory values are summarized in Table 1. The chest x-ray as seen in Fig. 1 showed clear lungs and no pleural effusions. The patient was hospitalized and placed on a nasal cannula and started on prednisone $20 \mathrm{mg}$ BID on Day 1 of admission for 3 days followed by prednisone $20 \mathrm{mg}$ orally daily for an additional 3 days. On Day 6 of hospitalization, the hospital course was complicated by atrial fibrillation with rapid ventricular response, treated with intravenous (IV) diltiazem. At that time, the patient was started on therapeutic Lovenox and also remdesivir for 5 days and was later switched to methylprednisolone $40 \mathrm{mg}$ IV every 8 hours. The WBC count continued to trend upwards. The WBC trend during the entire hospitalization is shown in Fig. 2. A peripheral blood smear showed many mature lymphocytes with multiple smudge cells and no blasts. Peripheral blood flow cytometry showed: "Chronic lymphocytic leukaemia/small lymphocytic lymphoma: $91 \%$ of the total". The patient's respiratory status continued to improve, and she was hospitalized for 13 days and subsequently discharged to subacute rehabilitation in a stable condition.

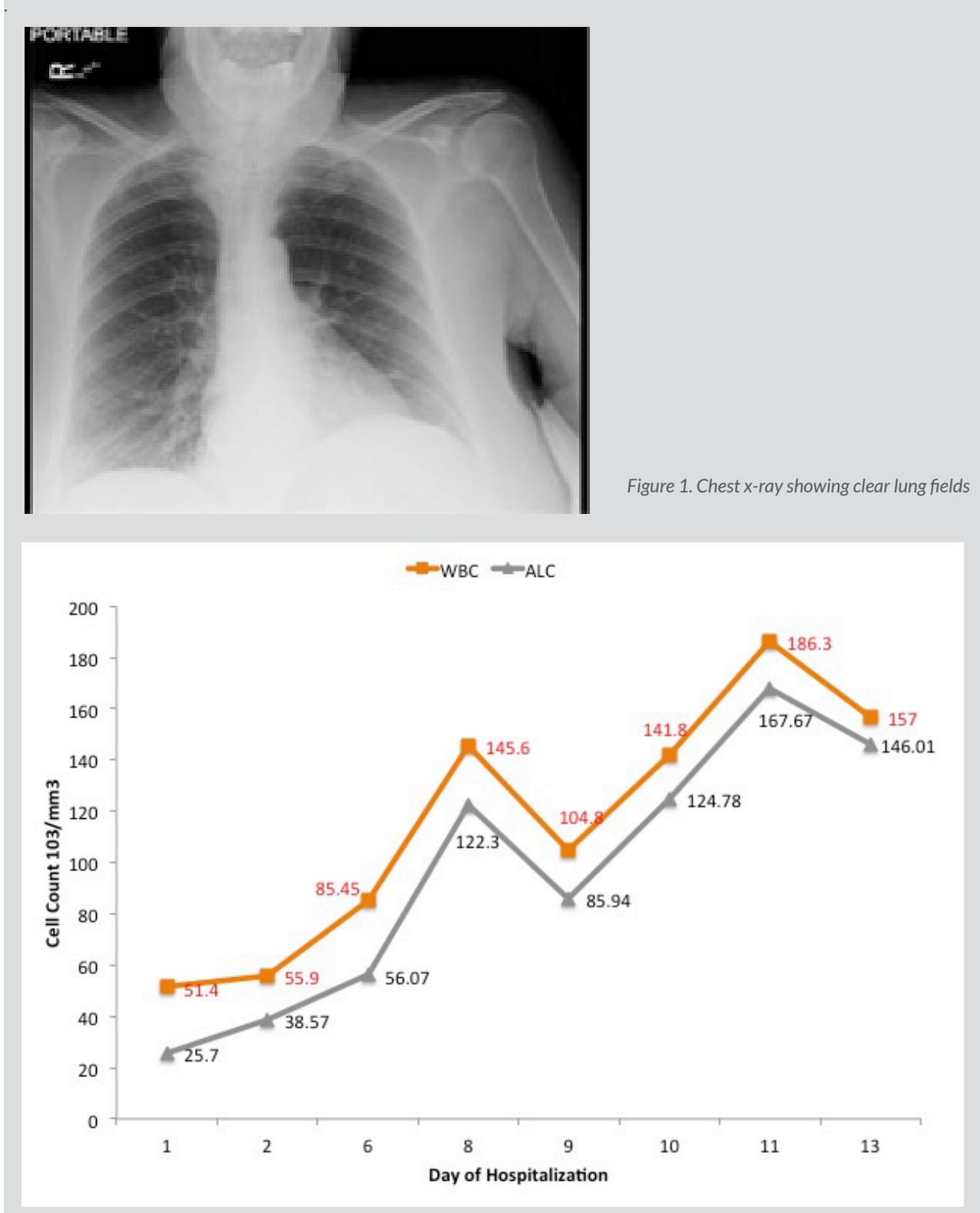

Figure 2. Graph showing the white blood cell (WBC) count and absolute lymphocyte count (ALC) corresponding to the day of hospitalization 


\section{CASE DESCRIPTION 2}

We report the case of a 79-year-old female, with a past medical history significant for CLL, hypertension and hypothyroidism, who presented to the ED complaining of fever, shortness of breath, dry cough and fatigue for 2 days. The symptoms were associated with intermittent fevers relieved by Tylenol, nasal congestion with discharge and dizziness. The patient was diagnosed with CLL in April 2013 and was under observation. The patient baseline counts were WBC $120 \mathrm{~K}$. She denied any chest pain, nausea, vomiting or diarrhoea but reported positive exposure to COVID-19. The nasopharyngeal swab tested positive in the SARS-CoV-2 (rRT-PCR) assay.

In the ED, initial vital signs were significant for temperature $36.9^{\circ} \mathrm{C}$, heart rate 94 beats/minute, respiratory rate 24 breaths/minute, blood pressure $114 / 64 \mathrm{mmHg}, \mathrm{SpO}_{2} 88 \%$ on room air. The physical examination was unremarkable. Relevant laboratory data are listed in Table 1. The chest $x$-ray as seen in Fig. 3 showed bilateral hazy infiltrates throughout both lung fields. The patient was initially placed on a nasal cannula and was started on azithromycin, ceftriaxone, dexamethasone and remdesivir. On Day 5 of hospitalization, the patient was noted to be in respiratory distress, and she was intubated and transferred to the intensive care unit (ICU) and managed for acute hypoxic respiratory failure and ARDS. The trend with respect to the WBC count and the absolute lymphocyte count is shown in Fig. $4 . \mathrm{On}$ Day 10 of hospitalization, the patient self-extubated. At that time, she was placed on a nonrebreather and oxygen saturation was approximately $96 \%$. The patient's condition remained stable throughout and she was discharged to home on Day 24 of hospitalization.

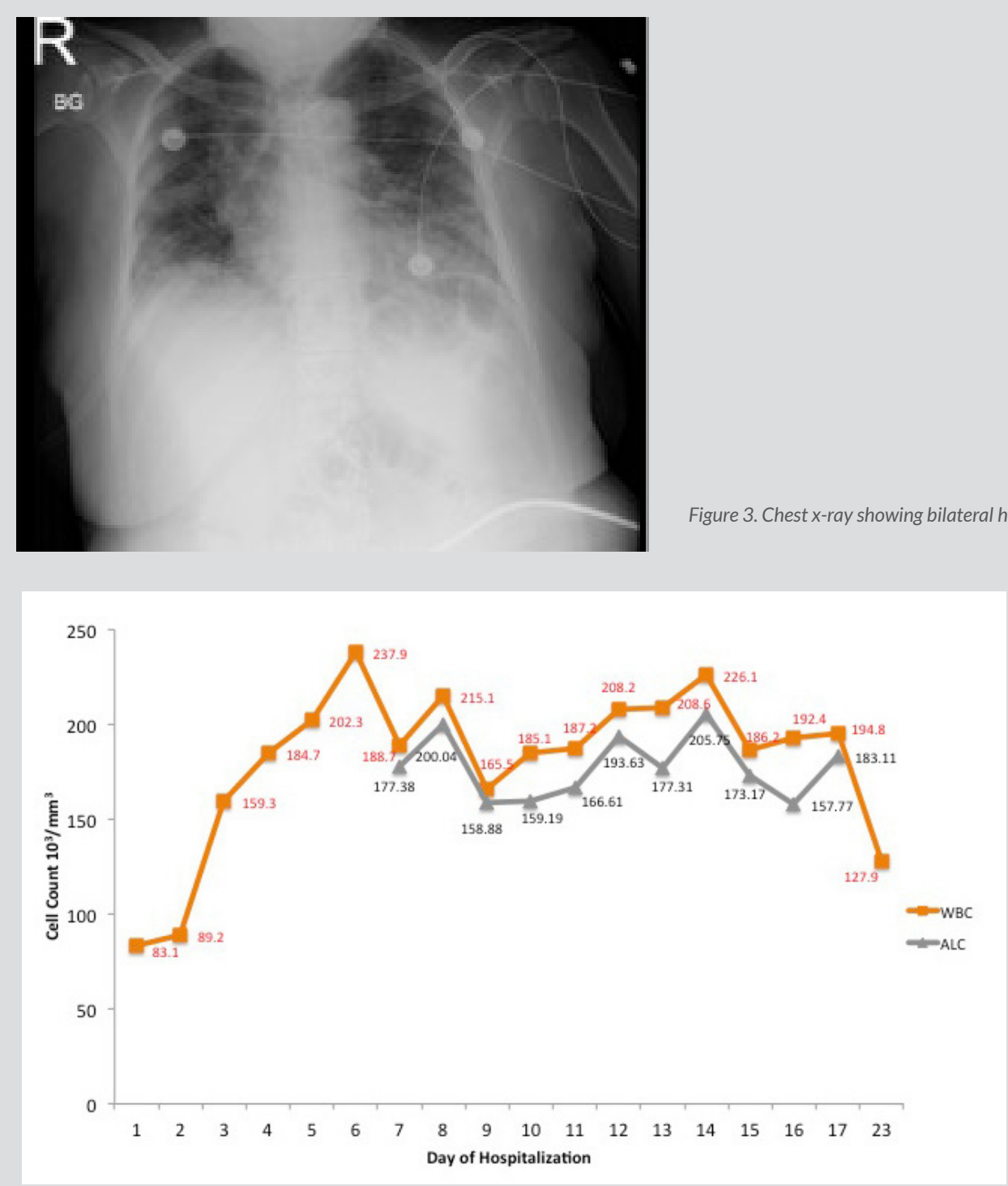

Figure 4. Graph showing the white blood cell (WBC) count and absolute lymphocyte count (ALC) corresponding to the day of hospitalization 


\section{of Case Reports in \\ Internal Medicine}

\begin{tabular}{|l|l|l|l|}
\hline Variable & Reference range & Case 1 & Case 2 \\
\hline White blood cell count & $4.5-11.0 \times 103 / \mathrm{mm}^{3}$ & 51.4 & 83.1 \\
\hline Haemoglobin & $13.5-17.5 \mathrm{~g} / \mathrm{dl}$ & 12 & 7.9 \\
\hline Platelets & $140-440 \mathrm{~K} / \mathrm{mm}^{3}$ & 181 & 210 \\
\hline Absolute lymphocyte count & $0.90-4.60 \times 103 / \mathrm{mm}^{3}$ & 25.7 & 64.82 \\
\hline Absolute neutrophil count & $1.30-7.80 \times 103 / \mathrm{mm}^{3}$ & 24.67 & 18.28 \\
\hline Basophils & $0-2 \times 103 / \mathrm{mm}^{3}$ & 0 & 0 \\
\hline Eosinophils & $0-5 \times 103 / \mathrm{mm}^{3}$ & 0 & 0 \\
\hline Reticulocyte count & $0.50-2.0 \%$ & $1.88 \%$ & $0.64 \%$ \\
\hline Direct antiglobulin test & Negative & Negative & Negative \\
\hline Haptoglobin & $42-346 \mathrm{mg} / \mathrm{dl}$ & 477 & 614 \\
\hline Ferritin & $16.4-294.0 \mathrm{ng} / \mathrm{ml}^{\prime}$ & 192 & 146 \\
\hline C-reactive protein (CRP) & $\leq 9.9 \mathrm{mg} / \mathrm{l}$ & 57.7 & 362.4 \\
\hline Lactate dehydrogenase (LDH) & $140-271 \mathrm{unit} / \mathrm{l}$ & 204 & 215 \\
\hline D-dimer & $\leq 0.50 \mathrm{mcg} / \mathrm{ml}$ & 2.37 & 1.53 \\
\hline Erythrocyte sedimentation rate (ESR) & $<10 \mathrm{~mm} / \mathrm{h}$ & 18 & 131 \\
\hline Immunoglobulin G (IgG) & $635-1,471 \mathrm{mg} / \mathrm{dl}$ & Not done & $\begin{array}{l}1,034 \mathrm{mg} / \mathrm{dl} \\
\text { (on Day 3 of hospitalization) }\end{array}$ \\
\hline Immunoglobulin M (IgM) & $40-280 \mathrm{mg} / \mathrm{dl}$ & $\begin{array}{l}35 \mathrm{mg} / \mathrm{dl} \\
\text { (on Day 3 of hospitalization) }\end{array}$ \\
\hline Immunoglobulin A (IgA) & $66-433 \mathrm{mg} / \mathrm{dl}$ & $\begin{array}{l}249 \mathrm{mg} / \mathrm{dl} \\
\text { (on Day 3 of hospitalization) }\end{array}$ \\
\hline
\end{tabular}

Table 1. Significant laboratory values at presentation to the ED

\section{DISCUSSION}

CLL is the most common leukaemia in adults in the Western world and is categorized by the presence of the proliferation of mature clonal B cells in the bloodstream, bone marrow, lymph nodes and spleen, which is confirmed by flow cytometry. A majority (80\%) of patients are asymptomatic at the time of diagnosis ${ }^{[5]}$.

The SARS-CoV-2 pandemic has led to profound morbidity and mortality around the globe. The outcome and disease course of COVID-19 in patients with haematological malignancies are not fully known. Mato et al. analyzed 198 patients with CLL and confirmed symptomatic COVID-19 in a multicentre study and concluded that irrespective of disease phase or status of treatment, CLL patients who were admitted are at high risk of death ${ }^{[6]}$. Lymphopenia has been associated with severe disease and is a poor prognostic factor in COVID-19-infected patients ${ }^{[7]}$. Vardanyan et al. reported the case of a 61-year-old patient with CLL who was diagnosed with COVID-19 and experienced a spontaneous partial resolution of CLL lymphocytosis for a short period ${ }^{[8]}$. However, contrary findings have also been reported; Paneesha et al. reported a case series of 4 CLL patients with COVID-19 with COVID-19-induced lymphocytosis ${ }^{[9]}$. Hyperleucocytosis is defined as a WBC count $>100 \times 10^{9} / \mathrm{l}(100,000 / \mu \mathrm{l})^{[10]}$. Leucostasis (also called symptomatic hyperleucocytosis) is a medical emergency and results from aggregation of leukaemic cells in the intravascular system, resulting in hypoxia and tissue injury ${ }^{[10]}$. Leucostasis is very rare in CLL and is more commonly seen in acute leukaemia ${ }^{[10]}$.

Anaemia in COVID-19 could be due to multiple reasons; anaemia related to inflammation or bleeding due to anticoagulation ${ }^{[11]}$. Patients with CLL/SLL have an increased incidence of autoimmune haemolytic anaemia (AIHA). The direct antiglobulin (Coombs) test (DAT) may be positive in up to $35 \%$ of cases at some time during the course of the disease; overt AlHA occurs in approximately $10 \%$ of cases, usually later in the disease course ${ }^{[12]}$. There have been reports of AIHA associated with COVID-19 ${ }^{[13]}$. Clinicians should be hypervigilant with CLL patients with COVID-19 regarding haemolysis. Haemolytic work-up was negative in our case series.

In addition to advanced age seen in CLL, these patients are considered immunocompromised due to dysregulation of humoral immunity as well as cellular immunity, and are thus more prone to developing infections ${ }^{[14,15]}$. This dysregulation is suggested by the presence of hypogammaglobulinaemia, and an impairment in the number and function of B and T cells ${ }^{[6]}$. Ye et al. reported a case of a 72 -year-old female with a history of lymphocytosis of unknown aetiology who was admitted to hospital after developing COVID-19 as part of a household 
cluster. The patient was diagnosed with COVID-19 and newly diagnosed CLL. Compared to her family members, the patient suffered from severe pneumonia (PNA) with superimposed bacterial infection; however, she recovered and was discharged approximately 20 days after admission. The authors concluded that early intervention with antivirals and high-dose IV immunoglobulin (IVIG) appeared to be effective in this patient ${ }^{[16]}$.

Langerbeins et al. reported a case of a 52-year-old male with multiple comorbidities and CLL who was admitted and managed in the ICU for COVID-19 PNA complicated by concomitant parainfluenza virus 4 infection. He was managed conservatively with high flow oxygen via a nasal cannula. The patient also received $30 \mathrm{~g}$ immunoglobulin G daily (IVIG) from Day 17 to Day 20. The patient was discharged on Day 28. The authors urged clinicians to conduct a complete examination of respiratory samples to rule out bacterial, viral and fungal superinfections in these patients and to administer immunoglobulins as per guidelines ${ }^{[17]}$.

Alves Barbosa et al. reported a case of a patient with CLL and SARS-CoV- 2 infection with a WBC count of $323 \times 10^{9}$ cells/l. The authors observed a discrepancy between persistently low measured $\mathrm{PaO} 2$ levels on $\mathrm{ABG}$ analysis and normal oxygen saturation. The authors drew attention to leucocytosis-induced pseudohypoxaemia due to increased consumption of dissolved oxygen in blood samples ${ }^{[18]}$.

Finally, the decision to continue, or discontinue, antileukaemic therapy should be made on an individual basis. FDA-approved therapy for CLL includes the use of Bruton tyrosine kinase inhibitors (BTKis) such as ibrutinib; purine analogues such as fludarabine and pentostatin; the anti-CD20 monoclonal antibodies obinutuzumab, ofatumumab and rituximab and alkylating agents such as bendamustine, chlorambucil and cyclophosphamide ${ }^{[5]}$. The BTK pathway is crucial for the development of several pro-inflammatory cytokines; thus, inhibiting this process leads to decreased release of cytokines and results in an anti-inflammatory effect. Thus, inhibition of BTK may represent a potential mechanism to reduce the excessive inflammation/immune response produced by SARS-CoV- $2^{[19]}$.

Several recent case series and reports support the possible protective effects of BTKis; however, there are still insufficient data to recommended continuing or discontinuing BTKis. In a recent study by Reda et al., the authors analyzed data from 2,902 patients with CLL, out of which 278 were on ibrutinib and 23 were noted to have confirmed SARS-CoV-2 infection. Among patients on ibrutinib, 4 patients were reported to have confirmed SARS-CoV-2 infection. One patient was managed as an outpatient while 3 patients developed ARDS with 2 requiring mechanical intubation. The patient with the highest burden of comorbidities died from COVID-19 complications. While the sample size was small, the authors speculated that perhaps the low prevalence (4/278) of COVID-19 amongst those on ibrutinib was due to its modulatory actions on the cell-mediated immune response and cytokine release ${ }^{[20]}$. Thibaud et al., in a cohort of 8 patients with CLL and COVID-19, observed that 2 patients who were continued on ibrutinib were characterized by a shorter hospitalization length, minimal oxygen requirements, and eventually, full recovery ${ }^{[19]}$. Lin et al. reported a case of a patient with CLL who was on ibrutinib and who then developed severe COVID-19 infection requiring mechanical ventilation. Ibrutinib was continued and the patient was extubated after 9 days and eventually discharged on room air, and the authors suggest that ibrutinib may have a protective effect by decreasing inflammation and preventing progression to ARDS [21].

\section{CONCLUSION}

While some studies concluded that the overall prevalence of COVID-19 in patients with CLL is not substantially higher than the average population, the fact remains that these patients are at increased risk of acquiring infections due to the inherent role of an impaired immune response, and thus, care needs to be taken by both clinicians and patients to prevent infection.

Therapy in individuals with CLL should be personalized, based on the overall medical history and clinical status. In conclusion, we report 2 cases of COVID-19-induced hyperleucocytosis (WBC count $>100,000 / \mu \mathrm{l}$ ) in CLL patients and further studies, perhaps randomized, are essential to examine the outcome of COVID-19 in patients with CLL and to clarify the role of BTKi therapy at the time of the pandemic. 


\section{REFERENCES}

1. World Health Organization (WHO) [Internet]. Coronavirus disease (COVID-19) dashboard. Available from: https://covid19.who.int/ (accessed 18 January 2021).

2. Pollard CA, Morran MP, Nestor-Kalinoski AL. The COVID-19 pandemic: a global health crisis. Physiol Genomics 2020;52(11):549-557.

3. Machhi J, Herskovitz J, Senan AM, Dutta D, Nath B, Oleynikov MD, et al. The natural history, pathobiology, and clinical manifestations of SARS-CoV-2 infections. J Neuroimmune Pharmacol 2020;15(3):359-386.

4. Liang W, Guan W, Chen R, Wang W, Li J, Xu K, et al. Cancer patients in SARS-CoV-2 infection: a nationwide analysis in China. Lancet Oncol 2020;21(3):335-337.

5. Charra B, Ellouadghiri A, Magramane A, Kebbou T, Damaan K, Maghfour A, et al. COVID-19 and fortuitous discovery of chronic lymphocytic leukemia: biological findings and therapeutic challenges. Pan Afr Med J 2020;36:286.

6. Mato AR, Roeker LE, Lamanna N, Allan JN, Leslie L, Pagel JM, et al. Outcomes of COVID-19 in patients with CLL: a multicenter international experience. Blood 2020;136(10):11341143.

7. Wang K, Zuo P, Liu Y, Zhang M, Zhao X, Xie S, et al. Clinical and laboratory predictors of in-hospital mortality in patients with coronavirus disease-2019: a cohort study in Wuhan, China. Clin Infect Dis 2020;71(16):2079-2088.

8. Vardanyan R, Arjomandi Rad A, Wilson F. Chronic lymphocytic leukaemia in COVID-19. Int J Lab Hematol 2020;42(6):e263-e265.

9. Paneesha S, Pratt G, Parry H, Moss P. Covid-19 infection in therapy-naive patients with B-cell chronic lymphocytic leukemia. Leuk Res 2020;93:106366.

10. Singh N, Singh Lubana S, Dabrowski L, Sidhu G. Leukostasis in chronic lymphocytic leukemia. Am J Case Rep 2020;21:e924798.

11. Algassim AA, Elghazaly AA, Alnahdi AS, Mohammed-Rahim OM, Alanazi AG, Aldhuwayhi NA, et al. Prognostic significance of hemoglobin level and autoimmune hemolytic anemia in SARS-CoV-2 infection. Ann Hematol 2021;100(1):37-43.

12. DiehI LF, Ketchum LH. Autoimmune disease and chronic lymphocytic leukemia: autoimmune hemolytic anemia, pure red cell aplasia, and autoimmune thrombocytopenia. Semin Oncol 1998;25(1):80-97.

13. Sahu KK, Borogovac A, Cerny J. COVID-19 related immune hemolysis and thrombocytopenia. J Med Virol 2021;93(2):1164-1170.

14. Scarfò L, Chatzikonstantinou T, Rigolin GM, Quaresmini G, Motta M, Vitale C, et al. COVID-19 severity and mortality in patients with chronic lymphocytic leukemia: a joint study by ERIC, the European Research Initiative on CLL, and CLL Campus. Leukemia 2020;34(9):2354-2363.

15. Ali E, Badawi M, Abdelmahmuod E, Kohla S, Yassin MA. Chronic lymphocytic leukemia concomitant with COVID 19: a case report. Am J Case Rep 2020;21:e926062.

16. Ye X, Xiao X, Li B, Zhu W, Li Y, Wu J, et al. Low humoral immune response and ineffective clearance of SARS-Cov-2 in a COVID-19 patient with CLL during a 69-day follow-up. Front Oncol 2020;10:1272.

17. Langerbeins P, Fürstenau M, Gruell H, Klein F, Persigehl T, Rybniker J, et al. COVID-19 complicated by parainfluenza co-infection in a patient with chronic lymphocytic leukemia. Eur J Haematol 2020;105(4):508-511.

18. Alves Barbosa O, Guimarães Andrade T, de Almeida Sousa MD, Correia JW. COVID-19 in a patient with chronic lymphocytic leukaemia with pseudohypoxemia. Eur J Case Rep Intern Med 2020;7(7):001763.

19. Thibaud S, Tremblay D, Bhalla S, Zimmerman B, Sigel K, Gabrilove J. Protective role of Bruton tyrosine kinase inhibitors in patients with chronic lymphocytic leukaemia and COVID-19. Br J Haematol 2020;190(2):e73-e76.

20. Reda G, Noto A, Cassin R, Zamprogna G, Borella C, Scarfò L, et al.; Rete Ematologica Lombarda (REL) Clinical Network. Reply to "CLL and COVID-19 at the Hospital Clinic of Barcelona: an interim report" Analysis of six hematological centers in Lombardy: on behalf of CLL commission of Lombardy Hematology Network (REL). Leukemia 2020;34(9):2531-2532.

21. Lin AY, Cuttica MJ, Ison MG, Gordon LI. Ibrutinib for chronic lymphocytic leukemia in the setting of respiratory failure from severe COVID-19 infection: case report and literature review. EJHaem. Epub 2020 Sep 20. doi: 10.1002/jha2.98. 